\title{
Adherence to Exercise Programs and Determinants of Maintenance in Older Adults With Mild Cognitive Impairment
}

\author{
Erwin C.P.M. Tak, Jannique G.Z. van Uffelen, \\ Mai J.M. Chin A Paw, Willem van Mechelen, \\ and Marijke Hopman-Rock
}

\begin{abstract}
After a randomized controlled trial showing that improvement on some aspects of cognitive function was related to adherence to an exercise program, determinants of adherence and maintenance were further studied. Older adults with mild cognitive impairment were contacted 6 mo after the end of exercise programs for a telephone interview addressing patterns of adherence and determinants of maintenance. Mean adherence during the trial was $53 \%$. About one third of participants had lapses during the trial but completed, one third had no lapses, and one third dropped out or never started. Practical barriers (time, location) were related to not starting and functional limitations to dropout. After the trial $25 \%$ of participants continued the programs, $14 \%$ reported intention to continue, and $61 \%$ quit. Maintenance was determined by fewer health complaints, higher satisfaction with the programs, and better adherence during the programs. Although maintenance was low, this study identified several reasons and barriers to adherence and maintenance that could be addressed.
\end{abstract}

Keywords: physical activity, aging, barriers, patterns of adherence

Regular physical activity and exercise have been widely associated with a variety of health benefits in older adults (Chodzko-Zajko et al., 2009; Cress et al., 2005) and also prevent and reduce the risk of developing secondary health problems in older adults with chronic conditions (Macera, Hootman, \& Sniezek, 2003).

Physical activity and exercise have also been reported to be associated with better cognitive status. According to prospective observational studies, being physi-

Tak is with the Dept. of Health Promotion, TNO Quality of Life, Leiden, The Netherlands. van Uffelen is with the School of Human Movement Studies, University of Queensland, Brisbane, Australia. Chin A Paw and van Mechelen are with the Dept. of Public and Occupational Health/EMGO Institute for Health and Care Research (EMGO+), VU University Medical Center, Amsterdam, The Netherlands. Hopman-Rock is with Body@Work, Research Center Physical Activity, Work and Health, TNO-VU University Medical Center, Amsterdam, The Netherlands. 
cally active not only prevents cognitive decline in healthy community-dwelling older women (Yaffe, Barnes, Nevitt, Lui, \& Covinsky, 2001) but also results in a reduced risk of cognitive impairment and any type of dementia 5 years later (Laurin, Verreault, Lindsay, MacPherson, \& Rockwood, 2001). Furthermore, a meta-analysis of exercise interventions concluded that aerobic fitness training improves some domains of cognitive function (i.e., executive-control processes) in healthy sedentary older adults, depending on length and type of intervention and duration of the training sessions (Colcombe \& Kramer, 2003). In another meta-analysis of older adults with dementia and cognitive decline, it was concluded that exercise training (most included studies used walking) had a positive effect on physical, cognitive, functional, and behavioral outcomes (Heyn, Abreu, \& Ottenbacher, 2004). Likewise, the cognitive status of older adults with mild cognitive impairment (MCI; Petersen et al., 1999) may improve, as well, if they participate regularly in aerobic exercise (Lautenschlager et al., 2008; van Uffelen, Chin A Paw, van Mechelen, \& Hopman-Rock, 2008).

Despite the health-enhancing benefits of physical activity, initiating exercise programs and adhering to them is often problematic for older adults. For those starting with structured exercise, about 10-50\% of participants drop out during the first 6 months, with most relapses occurring during the first 3 months (Dishman \& Sallis, 1994; Stiggelbout, Hopman-Rock, Tak, Lechner, \& Van Mechelen, 2005). Stiggelbout et al. (Stiggelbout, Hopman-Rock, Crone, Lechner, \& Van Mechelen, 2006; Stiggelbout et al., 2005) evaluated dropout and predictors of maintenance in healthy older adults participating in different types of exercise programs. Predictors of maintenance to exercise programs 6 months after the start were having short lapses or no lapses, a high intention to start exercise at baseline, a high perceived quality of the program, and a positive attitude toward exercise and some practical obstacles including bad weather and being bored with the program. Known barriers for exercise maintenance include poor health, physical limitations, female gender, lack of exercise experience, and several psychological factors such as low self-efficacy and external locus of control (Rhodes, Martin, Taunton, Donelly, \& Elliot, 1999; Schutzer \& Graves, 2004).

Little is known about adherence and maintenance in older adults with MCI who participate in structured exercise programs. The results of the Folate Physical Activity Cognition Trial (FACT), a randomized controlled trial examining the effect of moderate-intensity walking and low-intensity physical activity on cognitive function (van Uffelen, Hopman-Rock, Chin A Paw, \& van Mechelen, 2005), showed an interaction between the effect of the exercise program and adherence (van Uffelen et al., 2008). In women each percentage increase in session attendance to the walking program was associated with a significant improvement in performance on two cognitive tests. In men, there was no interaction, but session attendance of $75 \%$ or more in the walking program was associated with improved delayed recall (one test out of seven), compared with the activity program.

Therefore, information on reasons for dropout, for having lapses, and on barriers to maintenance can be vital for sustaining the effect of a walking program for this population and could also provide essential help for successfully implementing exercise programs in general. It was decided to follow up on participation in both exercise programs with an additional 6 months focusing entirely on adherence 
and maintenance to these two exercise programs (walking program and placebo low-intensity program).

The purposes of the current study were to determine the level of participation, adherence, and maintenance of the exercise programs in older adults with MCI during the 12-month trial and 6 months after its end; describe reasons and barriers for participation, adherence, and lapses; and identify determinants of maintenance 6 months after the end of the trial.

\section{Method}

\section{Study Design}

The FACT study was designed as a randomized placebo-controlled trial (RCT) to examine the effects of a moderate-intensity aerobic walking program (MIWP) on cognitive function of community-dwelling older adults with MCI. A nonaerobic low-intensity activity program (LI-AP) was added as a placebo exercise program. The study protocol has been described in detail elsewhere (van Uffelen et al., 2005) and was approved by the VU University Medical Center medical ethics committee. Written informed consent was obtained from all participants. For the follow-up study presented in this article, participants were interviewed about their physical activity and exercise behavior 6 months after the end of the 12-month exercise programs of the RCT. For this article, the distinction between the MI-WP and LI-AP programs as intervention versus control group was no longer relevant. Adherence and maintenance were studied for participants in both groups together.

\section{Participants}

Recruitment of the participants has been described in detail elsewhere (van Uffelen, Chin A Paw, Klein, van Mechelen, \& Hopman-Rock, 2007). In short, all community-dwelling adults age $70-80$ years $(N=5,491)$ in a medium-sized Dutch town were approached by mail. From the adults willing to participate, participants with MCI were identified using a two-step screening consisting of a postal questionnaire and a telephone interview in which the following inclusion criteria were assessed: self-reported memory complaints, no report of disability in activities of daily living as measured with the Groningen Activity Restriction Scale (Kempen, Miedema, Ormel, \& Molenaar, 1996), objective memory impairment as measured with a Dutch version of the 10-word learning test (Morris et al., 1989), and normal cognitive function and absence of dementia as assessed by the Telephone Interview for Cognitive Status (Brandt, Spencer, \& Folstein, 1988). In addition, participants completed the Mini Mental State Examination (MMSE) in a face-to-face interview, and those with an MMSE score $<24$ points were excluded.

After baseline measurements, included participants $(N=179)$ were randomly assigned to the MI-WP or LI-AP group. Randomization was stratified by baseline physical activity level in minutes per day. All participants who participated in the 12-month follow-up measurement of the RCT $(n=138)$ were invited for a telephone interview 6 months later focusing on participation, adherence, and maintenance for both program activities and habitual physical activity. 


\section{Interventions}

Both the MI-WP program and the LI-AP program were group based and lasted 12 months (June 2004 to June 2005). The frequency was twice a week and session duration was $60 \mathrm{~min}$. Sessions were supervised by qualified and trained instructors (van Uffelen, Chin A Paw, Hopman-Rock, \& van Mechelen, 2009).

The MI-WP was based on Sportive Walking, an existing aerobic walking program (Stahl \& Laukkanen, 2000). Intensity was moderate (>3 metabolic equivalents [METs]), and the aim was to improve aerobic fitness. Each session consisted of a warm-up, moderate-intensity walking exercise, and a cooldown. The intensity of walking in the MI-WP was increased gradually during the program by increasing the walking pace and distance. The program was developed in such a way that all participants could perform the walking exercise at their own level but still walk in a group. This was achieved by using walking routes with the same start and endpoint. In this way the participants could stay together in a group on the same track and by covering the track more times during the session adjust their pace. Sessions took place outdoors in municipal parks.

The LI-AP mainly consisted of aspects of programs that are widely available to older adults in The Netherlands, for example, balance and flexibility training and exercises for improving posture during activities of daily living. The program consisted of an introduction, low-intensity ( $<3 \mathrm{METs}$ ) nonaerobic group exercises such as light range-of-motion movements and stretching, and a closing. The program was carried out in community centers. After the intervention period for the RCT, the exercise programs continued as part of the local community service. Participants could continue at their own initiative for a small fee.

\section{Measurements}

Demographic variables were collected using a postal screening questionnaire before randomization. Data on cognitive function were collected during the baseline interview using the MMSE (Folstein, Folstein, \& McHugh, 1975) to assess general cognitive function. The Auditory Verbal Learning Test was used to assess memory (direct and delayed recall with maximum scores of 75 and 15 words, respectively; Rey, 1964). In this article, both instruments were used to describe the study population and as a possible determinant of maintenance. Physical activity levels were measured at baseline and after 6, 12, and 18 months using the LASA Physical Activity Questionnaire, which addresses the frequency and duration of housekeeping, sports activities, cycling, gardening, and walking during the previous 2 weeks (Stel et al., 2004). A summary measure per activity category was calculated by multiplying the number of hours per day with the appropriate MET from the "Compendium of Physical Activities" (Ainsworth et al., 1993) adapted for older adults (Verweij, Van Schoor, Deeg, Dekker, \& Visser, 2009).

Six months after the end of the exercise programs for the trial (T18), additional data on maintenance, determinants, and potential barriers for maintenance were collected from the participants during a structured telephone interview. Participants were asked to rate the general quality of the exercise programs and their satisfaction with 17 program-related aspects (Stiggelbout et al., 2006) on a 5-point Likert scale with a higher score indicating a better rating. These program-related aspects included instruction, attention, location, time, duration, other participants, intensity 
and frequency of the activity, effects on health, safety issues, and variation in the program. Dropout was defined as withdrawal before the end of the program at T12 as registered during the RCT. Adherence was defined as the proportion of attended sessions during the programs as registered by the instructors. Having lapses, defined as missing two consecutive sessions in a row, was evaluated by a single question during the structured telephone interview: "During the study, did you temporarily miss sessions of the walking or activity program?" Reasons for dropout and lapses were asked with one open-ended question: "If you temporarily missed sessions, please indicate the reason." Answers were later summarized into categories for presentation.

Finally, maintenance of the exercise program at T18 (continuing the exercise programs after the end of the RCT) was evaluated by a single question: "At the end of the study, did you continue with the program?" Participants were grouped as continued, intention to continue, or discontinued on the basis of their answers. Participants who discontinued were asked to evaluate 19 possible barriers (Stiggelbout et al., 2006) and indicate whether each was (1) very important, (2) important, (3) somewhat important, or (4) not important. Barriers included health complaints (injuries, limitations), practical aspects (time, location), whether progress was noted, program-related aspects (intensity, frequency), and personal aspects (interest in exercise, social contacts).

\section{Analysis}

All analyses were performed using SPSS for Windows (release 14.0). For descriptive purposes, demographic and cognitive variables at baseline were compared between the dropouts from the RCT and the participants who attended the follow-up measurements at T6 and T12 using Student's $t$ test for continuous variables and a chi-square test for categorical variables.

Second, to see if physical activity levels changed over time between MI-WP and LI-AP, a MANOVA with repeated measures was carried out.

The number of participants who started the programs, dropped out, or had lapses and the adherence were reported and compared between the MI-WP and LI-AP groups using chi-square analysis. Possible determinants for maintenance were compared between three groups-continued, intention, and discontinuedusing chi-square and univariate analysis of variance (ANOVA). A difference was considered statistically significant if $p<.05$.

\section{Results}

One hundred thirty-eight of the 179 participants who participated in the baseline measurement of the project FACT participated in the two follow-up measurements at T6 and T12, as well. One hundred thirty-four of them could be contacted and were willing to participate in the telephone interview 6 months after the end of the exercise programs (response rate 97\%). One participant had died, and 3 others could not be contacted. There were hardly any missing data, with a maximum of $2 \%$ of cases missing on some variables (indicated in results if relevant). Table 1 provides an overview of baseline characteristics for participants in the telephone interview ( $n=134)$ and participants lost to follow-up during the 12-month trial $(n=45)$. 


\section{Table 1 Baseline Characteristics of Participants and Dropouts} and of the Telephone Interview at T18

\begin{tabular}{lccc}
\hline & Total at T0 & Interview (T18) & Dropouts (T0-T12) \\
\hline Number of participants & 179 & 134 & 45 \\
MI-WP & $86 / 179$ & $71 / 134$ & $15 / 45$ \\
LI-AP & $93 / 179$ & $63 / 134^{*}$ & $30 / 45$ \\
Mean age (SD) & $75.1(2.9)$ & $74.8(2.9)^{*}$ & $75.9(2.6)$ \\
\% Female & 44 & 41 & 51 \\
Education (\%) & & & \\
$\quad$ low & 54 & 55 & 51 \\
$\quad$ middle & 25 & 28 & 18 \\
$\quad$ high & 18 & 17 & 20 \\
\% Living alone & 31.8 & $25.4 *$ & 51.1 \\
Mean MMSE (SD) & $28.3(1.5)$ & $28.4(1.4)$ & $28.0(1.5)$ \\
$\quad$ possible range (0-30) & $24-30$ & $24-30$ & $24-30$ \\
Mean AVLT direct recall & $32.5(8.1)$ & $33.1(7.9)$ & $30.9(8.4)$ \\
$\quad$ possible range (0-75) & $9-54$ & $15-54$ & $9-46$ \\
Mean AVLT delayed recall & $5.9(2.5)$ & $5.9(2.4)$ & $5.6(3.0)$ \\
$\quad$ possible range (0-15) & $0-12$ & $1-12$ & $0-11$ \\
\hline
\end{tabular}

Note. MI-WP $=$ moderate-intensity walking program; LI-AP = low-intensity activity program; MMSE $=$ Mini Mental State Examination; AVLT = Auditory Verbal Learning Test.

aConsist of 41 participants who did not attend the last follow-up measurement at 12 months and 4 participants who participated in all measurements but could not be contacted for the interview at 18 months (1 died, 3 not reached).

*Significantly different from dropouts, $p<.05$.

Participants lost to follow-up were older $(t=2.25, d f=177, p<.05)$, more likely to live alone (chi-square $=10.3, d f=1, p<.01$ ), and were more likely to be from the LI-AP group (chi-square $=5.2, d f=1, p<.05$ ) than the 134 participants who continued the trial and participated in the telephone interview.

Figure 1 displays the levels of different types of physical activity in MET values at T0, T6, T12, and T18 for the MI-WP and LI-AP groups separately. There was a significant decrease in overall habitual daily physical activity level $(F=7.94, p<$ .001 ) over time but no difference between groups.

\section{Participation in the Exercise Programs During the RCT}

Results from the telephone interview at T18, presented in Table 2, show that 30 of 134 participants did not start their designated exercise program (equally distributed over both programs). Main reasons for not starting were being too occupied with other activities (23\%), having trouble with walking or moving (23\%), estimating the intensity of the program as being too vigorous or too light (20\%), or being ill (17\%; Table 3). 


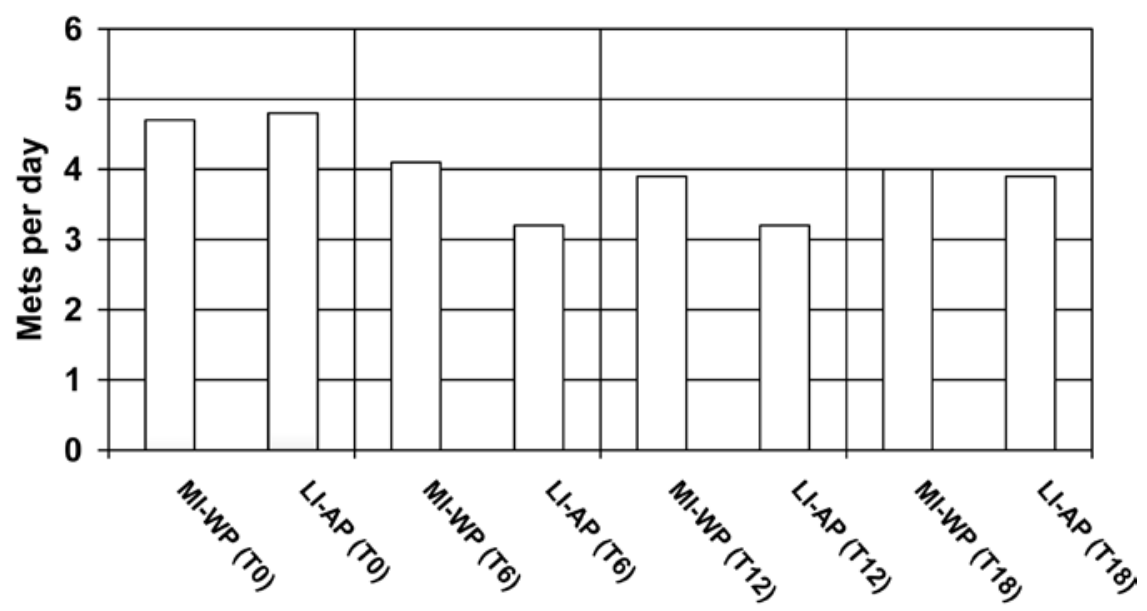

Figure 1 - Level of intensity in metabolic equivalents (METs) of habitual physical activity as measured with the LASA Physical Activity Questionnaire at four times (T0, T6, T12, and T18) for two exercise programs: a moderate-intensity walking program (MI-WP) and a low-intensity activity program (LI-AP).

Table 2 Participation, Adherence, and Maintenance in the Moderate-Intensity Walking Program (MI-WP) and Low-Intensity Activity Program (LI-AP), $n$ (\%)

\begin{tabular}{llccc}
\hline Stage & Status & $\begin{array}{c}\text { MI-WP } \\
(\boldsymbol{n}=68)\end{array}$ & $\begin{array}{c}\text { Ll-AP } \\
(\boldsymbol{n}=64)\end{array}$ & $\begin{array}{c}\text { Total } \\
(\mathbf{N}=132)^{\mathbf{a}}\end{array}$ \\
\hline Participation & Started & $52(76 \%)$ & $50(78 \%)$ & $102(77 \%)$ \\
& Not started & $16(24 \%)$ & $14(22 \%)$ & $30(23 \%)$ \\
Adherence & Finished without lapses & $25(48 \%)$ & $26(52 \%)$ & $51(50 \%)$ \\
& Finished with lapses & $17(33 \%)$ & $21(42 \%)$ & $38(37 \%)$ \\
& Dropped out & $10(19 \%)$ & $3(6 \%)$ & $13(13 \%)$ \\
Maintenance & Mean \% attendance (SD) & $49(33.9)$ & $55(36.3)$ & $53(34.8)$ \\
& Continued & $18(26 \%)$ & $19(29 \%)$ & $37(28 \%)$ \\
& Intention to continue & $14(20 \%)$ & $5(8 \%)$ & $19(14 \%)$ \\
& Discontinued & $37(54 \%)$ & $41(63 \%)$ & $78(58 \%)$ \\
\hline
\end{tabular}

aData from 2 participants missing.

\section{Adherence to the Exercise Programs During the RCT}

Of the 104 participants who started one of two exercise program, data from 2 participants were missing. Of the 102 remaining, 13 dropped out during the program (10 in MI-WP, 3 in LI-AP; Table 2). Main reasons for dropping out were having trouble with walking or moving and being ill or injured (not related to the program). 
Table 3 Reasons for Not Starting the Program, Dropping Out, and Having Lapses for the Two Programs (Number of Participants Who Mentioned the Reason)

\begin{tabular}{llccc}
\hline \multirow{2}{*}{ Type } & Reason & $\begin{array}{c}\text { Never } \\
\text { started }\end{array}$ & $\begin{array}{c}\text { Dropped } \\
\text { out }\end{array}$ & Lapses \\
\hline Program related & Intensity too high & 4 & 1 & 0 \\
& Intensity too low & 2 & 0 & 0 \\
\multirow{5}{*}{ Practical } & Complaints related to program & 0 & 2 & 0 \\
& Too occupied & 7 & 1 & 0 \\
\multirow{5}{*}{ Physical } & Bad location & 1 & 0 & 0 \\
& Problems with walking or & 5 & 5 & 0 \\
\multirow{5}{*}{ Other } & moving & & & \\
& Unrelated illness or injury & 5 & 4 & 10 \\
& Holiday & 2 & 0 & 28 \\
& Not in preferred program & 1 & 0 & 0 \\
\multirow{3}{*}{ Total } & Doesn't need it & 2 & 0 & 0 \\
\hline
\end{tabular}

Two participants dropped out because they experienced complaints as a result of the exercise programs ( 1 out of each program). One participant judged the MI-WP program to be too vigorous.

Mean adherence rates during the 12-month exercise programs were around $50 \%$ and did not differ between exercise groups $(t=1.07, d f=132, p=.29)$. The 13 dropouts attended about one third of the sessions (34\%) before leaving the program.

Of the 89 participants who finished the total MI-WP or LI-AP program, 51 did not report any lapses, and 38 reported one or more lapses, because of either being on a holiday ( $74 \%$ ) or being ill or injured (26\%; not related to the program).

The number of participants who did not start, dropped out, or had lapses did not differ significantly between the two groups (chi-square $4.2, d f=3, p=.24$ ).

\section{Maintenance of the Programs After the RCT}

More than half $(n=78)$ of the 132 participants interviewed at T18 had discontinued participation in the exercise programs after the end of the trial, one quarter $(n=$ $37)$ continued, and one seventh $(n=19)$ intended to continue in the near future. Maintenance status did not differ significantly between the MI-WP and LI-AP groups (chi-square $=4.38, d f=2, p=.11$ ).

Health complaints (including injuries) and practical barriers (time and location) were the most frequently mentioned barriers to maintenance (see Table 4). In addition, personal reasons such as lack of interest ("don't feel like it"), lack of (subjective) progress, or the program not living up to their expectations were 
Table 4 Barriers to Continuing the Exercise Program

\begin{tabular}{lcc}
\hline Reason & $\begin{array}{c}\text { Percentage of participants } \\
\text { who mentioned the barrier }\end{array}$ & $\begin{array}{c}\text { Mean level of } \\
\text { importance }^{\mathrm{a}}(\mathbf{S D})\end{array}$ \\
\hline Health complaints & 36 & $3.1(1.2)$ \\
Lack of time & 35 & $3.2(1.2)$ \\
Injuries & 31 & $3.4(0.8)$ \\
Doesn't feel like it & 21 & $3.7(0.7)$ \\
Practical: time of day & 20 & $3.6(0.9)$ \\
No progress & 16 & $3.8(0.5)$ \\
Doesn't live up to expectation & 15 & $3.7(0.7)$ \\
Practical: location & 15 & $3.7(0.9)$ \\
Too tired & 15 & $3.8(0.7)$ \\
Intensity too high or low & 13 & $3.8(0.7)$ \\
Too expensive & 10 & $3.8(0.7)$ \\
Not used to making effort & 8 & $3.9(0.5)$ \\
No contact with others & 6 & $3.9(0.4)$ \\
Doesn't want to go alone & 5 & $3.9(0.4)$ \\
\hline
\end{tabular}

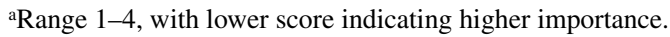

mentioned by $15-20 \%$ of the participants. Two barriers showed a statistically significant difference concerning importance rating between the groups. Participants in the MI-WP group ranked health complaints as a more important barrier than participants in the LI-AP group (mean 2.6 vs. 3.6, $t=3.969, d f=76, p<.001$ ), as they did being too tired (3.5 vs. 3.9, $t=2.56, d f=75, p=.01)$.

Finally, demographic, cognitive, adherence, and quality factors relevant to maintenance were compared between three status groups: participants who continued $(n=37)$, intended to continue $(n=19)$, or discontinued $(n=78)$. There were no significant between-groups differences in demographic factors, cognitive function at baseline, and type of program (Table 5).

The percentage of participants with lapses was significantly higher in the group that continued the exercise programs after the end of the RCT than in the other two groups. The highest number of dropouts during the RCT was found among those who discontinued. Attendance rates during the RCT were highest among those who continued, followed by those who intended to continue.

Although general satisfaction was not associated with maintenance status, other specific quality aspects were. Quality of the instructor and contents of the program were rated significantly lower in the intention and discontinue groups. Participants who did not continue evaluated the program as not being of the right intensity (most of them rating intensity as too high). Finally, participants who did not continue or only showed an intention to continue reported having experienced significantly less progress in their health or fitness level as a result of the program than did the group that continued. 


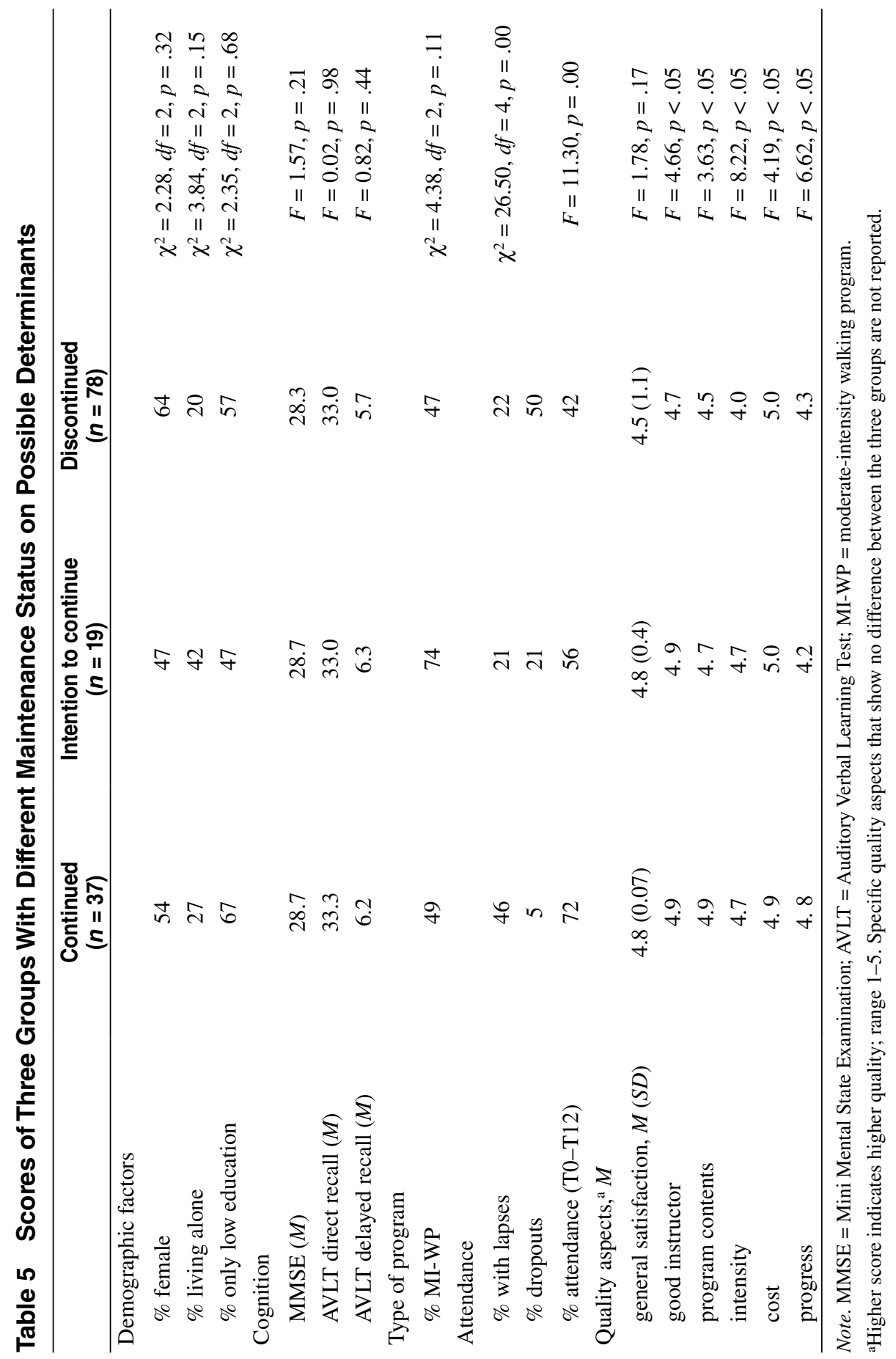




\section{Discussion}

This study showed that maintenance of participation in exercise programs in elderly adults with $\mathrm{MCI}$ is low; only $25 \%$ continued exercising after the end of the 12-month RCT. In addition, self-reported habitual physical activity levels were lower than preintervention levels, although seasonal effects cannot be ruled out.

There were differences in the reasons for starting, dropping out, having lapses, and maintenance for the two exercise programs, which calls for phase-specific interventions to improve compliance. Although not starting was mainly related to practical reasons, participants dropped out mainly because of physical limitations, and the most important barriers to maintenance were health complaints, practical limitations, and quality aspects of the exercise programs (instruction, contents, and intensity). Demographic and cognitive factors were not associated with maintenance. Lapses did not lead to dropout if they occurred because of a holiday or illness.

Adherence levels in this study varied $50-80 \%$ and were comparable to other group-based exercise programs lasting 12 months. Average adherence of older adults to exercise programs is reported to be around $75 \%$ (Van der Bij, Laurant, \& Wensing, 2002) but is known to decrease if dropouts are included (Martin \& Sinden, 2001). The number of dropouts from the MI-WP in our study sample with MCI was relatively low (13\%) and corresponded to dropout from Sportive Walking programs in the Netherlands, available to older adults outside a research setting (Stiggelbout et al., 2005). Most frequently mentioned reasons for dropping out in other studies are illness, pain, and lack of time or being too occupied (Martin \& Sinden, 2001), although in our study these were mainly related to physical problems in carrying out the program. This can explain why fewer participants dropped out of the less intensive LI-AP program.

About $25 \%$ of participants continued the exercise program after the end of the RCT, which is comparable to other programs for community-dwelling older adults (Hopman-Rock \& Westhoff, 2002). As is known from other studies, most important barriers to maintenance of exercise programs include health problems and practical limitations (Forkan et al., 2006; Rhodes et al., 1999). In this study quality aspects also played an important role in maintaining physical exercise. This overlaps with a previous study in which quality of the instructor and contents of the program were important in maintaining participation in exercise programs (Stiggelbout et al., 2006). Furthermore, participants in this study reported that the intensity of the program affected their maintenance. This was specifically the case in the MI-WP program. A potential way to avoid this in future studies could be to monitor and adjust the intensity of the exercise program (because this has been shown to be associated with motivation; Cress et al., 2005). Although participants were offered options to adjust exercise intensity in the MI-WP program, they were too limited for some of them. Ten participants mentioned cost being a barrier in the interview. Participation in the exercise program was free during the 12-month intervention. Although participation in the programs after the RCT was subsidized and participants only had to pay a small fee, it was still considered too expensive by some.

Our finding that determinants for starting, dropping out, and maintaining participation in exercise programs are different corresponds with other studies (van 
Stralen, Lechner, Mudde, de Vries, \& Bolman, 2008; Rothman, 2000); this suggests difference between determinants for initiating, adhering to, or maintaining exercise. Physical health status and a busy lifestyle are among the few reasons that determine both initiation and maintenance. This provides possibilities to address the most relevant barriers and determinants related to the specific phases of exercise behavior.

\section{Limitations of the Study}

We could only include participants who had completed the RCT and participated in all measurements. This means that participants in this extension study were a selective subgroup of the original. Comparisons between the dropouts and the adults who remained involved in the trial indeed showed differences; participants who were lost to follow-up were older and mainly in the LI-AP arm of the RCT, and a higher percentage of them lived alone. This means that the findings of this study may only be generalizable to older adults with memory complaints who are interested in starting exercise programs.

Data on the quality of the exercise programs, having lapses, and reasons for dropping out were gathered 6 months after the end of the main study. Because the population with MCI was defined by having memory problems, possible incorrect recollection should be taken into account. However, the median MMSE score indicated that participants had good general cognitive function, so it is not likely that this recall bias affected the results to a large extent.

Other relevant predictors of maintenance such as self-efficacy, attitude, and social support (McAuley, Jerome, Elavsky, Marquez, \& Ramsey, 2003) were not evaluated in this study to reduce the measurement burden on this population, but it is known from the literature that self-efficacy is a major determinant of physical activity (McAuley \& Blissmer, 2000), and it is likely not to be different in our study sample.

Exercise intensity differed between the MI-WP and the LI-AP. Because intensity can play a role in continuing the program, participants in the MI-WP could have been more prone to drop out than participants in the LI-AP before the follow-up measurement (at T18) and therefore may not have been included in the sample of the current study. Further research is therefore needed to investigate the critical balance between minimal intensity and duration and effectiveness, taking into account the increased dropout risk that is associated with exercise intensity.

Because the FACT study was designed as an RCT, people were randomized to a program that may not have been their program of choice. Although it was ensured that the program was run in the area where participants lived, they could not choose a specific time. This may have affected the results, because location and time affect participation. Only 1 person mentioned this as a reason to not start an exercise program, however, so it is not likely that this affected the results to a large extent.

This study adds to the current knowledge about behavioral aspects of physical activity promotion. Given the fact that most Western countries face aging populations and an associated increase in prevalence of age-related neurocognitive disorders, the specific focus on older adults with memory complaints living in the community is important. Improving their lifestyle by increasing their participation in physical activity and exercise can prevent functional decline, reduce the demand for health care, and prolong independent living until older age. 
Adherence was registered by instructors, but these data were not usable to measure lapses in adherence because they were collected as a percentage of attended sessions, rather than patterns of attendance. Therefore, we had to rely on self-report measures regarding lapses. Although we have no indication that self-report measures in this group with MCI were unreliable, future research should look into the possibility of using objective measures for physical activity, such as accelerometers. In addition, future studies should look into the possibility of monitoring patterns of adherence and consequently collect data on lapses during interventions.

\section{Conclusion and Recommendations}

Although it is not easy for older adults with MCI to maintain participation in exercise programs, this study shows there are different reasons and barriers that can be addressed. Practical aspects such as time and location of a program are important reasons for not starting, and these factors should be individually adjusted to prevent them from becoming actual barriers. Lapses because of holidays and illness do not jeopardize adherence, but dissatisfaction with the content (intensity) of the program and physical limitations do. Especially in moderate-intensity programs, such as an aerobic walking program, exercises tailored to the individual should be offered to prevent dropout and improve attendance. Individually tailored exercises could also be a solution for health complaints and limitations that prevent the elderly from participating in exercise programs. Because participants who reported clear benefits were more likely to continue participation in the exercise programs, feedback by instructors on subjective indicators could be worthwhile. Last but not least, providing a high-quality instructor and feasible content and addressing participants' specific outcome expectations could further enhance adherence and maintenance to obtain the highest possible health benefits.

\section{References}

Ainsworth, B.E., Haskell, W.L., Leon, A.S., Jacobs, D.R., Jr., Montoye, H.J., Sallis, J.F., \& Paffenbarger, R.S., Jr. (1993). Compendium of physical activities: Classification of energy costs of human physical activities. Medicine and Science in Sports and Exercise, 25, 71-80.

Brandt, J., Spencer, M., \& Folstein, M. (1988). The telephone interview for cognitive status. Neuropsychiatry, Neuropsychology, and Behavioral Neurology, 1, 111-117.

Chodzko-Zajko, W.J., Proctor, D.N., Fiatarone Singh, M.A., Minson, C.T., Nigg, C.R., Salem, G.J., \& Skinner, J.S. (2009). American College of Sports Medicine position stand. Exercise and physical activity for older adults. Medicine and Science in Sports and Exercise, 41, 1510-1530.

Colcombe, S., \& Kramer, A.F. (2003). Fitness effects on the cognitive function of older adults: A meta-analytic study. Psychological Science, 14, 125-130.

Cress, M.E., Buchner, D.M., Prohaska, T., Rimmer, J., Brown, M., Macera, C., . . ChodzkoZajko, W. (2005). Best practices for physical activity programs and behavior counseling in older adult populations. Journal of Aging and Physical Activity, 13, 61-74.

Dishman, R.K., \& Sallis, J.F. (1994). Determinants and interventions for physical activity and exercise. In C. Bouchard, R. Shephard, \& T. Stephens (Eds.), Physical activity, fitness, and health: International proceedings and consensus statement. Champaign, IL: Human Kinetics. 
Folstein, M.F., Folstein, S.E., \& McHugh, P.R. (1975). "Mini-Mental State.” A practical method for grading the cognitive state of patients for the clinician. Journal of Psychiatric Research, 12, 189-198.

Forkan, R., Pumper, B., Smyth, N., Wirkkala, H., Ciol, M.A., \& Shumway-Cook, A. (2006). Exercise adherence following physical therapy intervention in older adults with impaired balance. Physical Therapy, 86, 401-410.

Heyn, P., Abreu, B.C., \& Ottenbacher, K.J. (2004). The effects of exercise training on elderly persons with cognitive impairment and dementia: A meta-analysis. Archives of Physical Medicine and Rehabilitation, 85, 1694-1704.

Hopman-Rock, M., \& Westhoff, M.H. (2002). Development and evaluation of "Aging Well and Healthily": A health-education and exercise program for community-living older adults. Journal of Aging and Physical Activity, 10, 364-381.

Kempen, G.I., Miedema, I., Ormel, J., \& Molenaar, W. (1996). The assessment of disability with the Groningen Activity Restriction Scale. Conceptual framework and psychometric properties. Social Science \& Medicine, 43, 1601-1610.

Laurin, D., Verreault, R., Lindsay, J., MacPherson, K., \& Rockwood, K. (2001). Physical activity and risk of cognitive impairment and dementia in elderly persons. Archives of Neurology, 58, 498-504.

Lautenschlager, N.T., Cox, K.L., Flicker, L., Foster, J.K., van Bockxmeer, F.M., Xiao, J., . . . Almeida, O.P. (2008). Effect of physical activity on cognitive function in older adults at risk for Alzheimer disease: A randomized trial. Journal of the American Medical Association, 300, 1027-1037.

Macera, C.A., Hootman, J.M., \& Sniezek, J.E. (2003). Major public health benefits of physical activity. Arthritis Care and Research, 49, 122-128.

Martin, K.A., \& Sinden, A.R. (2001). Who will stay and who will go? A review of older adults' adherence to randomized controlled trials of exercise. Journal of Aging and Physical Activity, 9, 91-114.

McAuley, E., \& Blissmer, B. (2000). Self-efficacy determinants and consequences of physical activity. Exercise and Sport Sciences Reviews, 28, 85-88.

McAuley, E., Jerome, G.J., Elavsky, S., Marquez, D.X., \& Ramsey, S.N. (2003). Predicting long-term maintenance of physical activity in older adults. Preventive Medicine, $37,110-118$.

Morris, J.C., Heyman, A., Mohs, R.C., Hughes, J.P., van Belle, G., Fillenbaum, G., . . . Clark, C. (1989). The Consortium to Establish a Registry for Alzheimer's Disease (CERAD). Part I. Clinical and neuropsychological assessment of Alzheimer's disease. Neurology, 39, 1159-1165.

Petersen, R.C., Smith, G.E., Waring, S.C., Ivnik, R.J., Tangalos, E.G., \& Kokmen, E. (1999). Mild cognitive impairment: Clinical characterization and outcome. Archives of Neurology, 56, 303-308.

Rey, A. (1964). L'examen clinique en psychologie. Paris: Presses Universitaires de France.

Rhodes, R.E., Martin, A.D., Taunton, J.E., Donelly, M., \& Elliot, J. (1999). Factors associated with exercise adherence among older adults. An individual perspective. Sports Medicine (Auckland, N.Z.), 28, 397-411.

Rothman, A.J. (2000). Toward a theory-based analysis of behavioral maintenance. Health Psychology, 19, 64-69.

Schutzer, K.A., \& Graves, B.S. (2004). Barriers and motivations to exercise in older adults. Preventive Medicine, 39, 1056-1061.

Stahl, T., \& Laukkanen, R. (2000). A way of healthy walking - A guidebook for health promotion practice. Helsinki, Finland: The Finnish Rheumatism Association.

Stel, V.S., Smit, J.H., Pluijm, S.M.F., Visser, M., Deeg, D.J.H., \& Lips, P. (2004). Comparison of the LASA Physical Activity Questionnaire with a 7-day diary and pedometer. Journal of Clinical Epidemiology, 57, 252-258. 
Stiggelbout, M., Hopman-Rock, M., Crone, M., Lechner, L., \& Van Mechelen, W. (2006). Predicting older adults' maintenance in exercise participation using an integrated social psychological model. Health Education Research, 21, 1-14.

Stiggelbout, M., Hopman-Rock, M., Tak, E., Lechner, L., \& Van Mechelen, W. (2005). Dropout from exercise programs for seniors: A prospective cohort study. Journal of Aging and Physical Activity, 13, 406-421.

Van der Bij, A.K., Laurant, M.G.H., \& Wensing, M. (2002). Effectiveness of physical activity interventions for older adults-A review. American Journal of Preventive Medicine, 22, 120-133.

van Stralen, M.M., Lechner, L., Mudde, A.N., de Vries, H., \& Bolman, C. (2008). Determinants of awareness, initiation and maintenance of physical activity among the overfifties: A Delphi study. Health Education Research, 25, 233-247.

Van Uffelen, J.G.Z., Chin A Paw, M.J.M., Klein, M., Van Mechelen, W., \& Hopman-Rock, M. (2007). Detection of memory impairment in the general population: Screening by questionnaire and telephone compared to subsequent face-to-face assessment. International Journal of Geriatric Psychiatry, 22, 203-210.

Van Uffelen, J.G.Z., Chin A Paw, M.J.M., Hopman-Rock, M., \& van Mechelen W. (2009). Feasibility and effectiveness of walking program for community-dwelling older adults with mild cognitive impairment. Journal of Aging and Physical Activity, 17, 398-415.

Van Uffelen, J.G.Z., Chin A Paw, M.J.M., Van Mechelen, W., \& Hopman-Rock, M. (2008). Walking or vitamin B for cognition in older adults with mild cognitive impairment? A randomised controlled trial. British Journal of Sports Medicine, 42, 344-351.

Van Uffelen, J.G.Z., Hopman-Rock, M., Chin A Paw, M.J.M., \& Van Mechelen, W. (2005). Protocol for Project FACT: A randomised controlled trial on the effect of a walking program and vitamin B supplementation on the rate of cognitive decline and psychosocial wellbeing in older adults with mild cognitive impairment [ISRCTN19227688]. BMC Geriatrics, 5, 18. doi: 10.1186/1471-2318-5-18

Verweij, L.M., Van Schoor, N.M., Deeg, D.J., Dekker, J., \& Visser, M. (2009). Physical activity and incident clinical knee osteoarthritis in older adults. Arthritis and Rheumatism, 61, 152-157.

Yaffe, K., Barnes, D., Nevitt, M., Lui, L.Y., \& Covinsky, K. (2001). A prospective study of physical activity and cognitive decline in elderly women: Women who walk. Archives of Internal Medicine, 161, 1703-1708. 